APS/123-QED

\title{
Evolving Lorentzian wormholes supported by phantom matter and cosmological constant
}

\author{
Mauricio Cataldd* \\ Departamento de Física, Facultad de Ciencias, Universidad del Bío-Bío, \\ Avenida Collao 1202, Casilla 5-C, Concepción, Chile. \\ Sergio del Campd \\ Instituto de Física, Facultad de Ciencias, \\ Pontificia Universidad Católica de Valparaíso, \\ Avenida Brasil 2950, Valparaíso, Chile. \\ Paul Minning and Patricio Salgadd§ \\ Departamento de Física, Facultad de Ciencias Físicas y Matemáticas, \\ Universidad de Concepción, Casilla 160-C, Concepción, Chile.
}

(Dated: September 13, 2021)

\begin{abstract}
In this paper we study the possibility of sustaining an evolving wormhole via exotic matter made of phantom energy in the presence of a cosmological constant. We derive analytical evolving wormhole geometries by supposing that the radial tension of the phantom matter, which is negative to the radial pressure, and the pressure measured in the tangential directions have barotropic equations of state with constant state parameters. In this case the presence of a cosmological constant ensures accelerated expansion of the wormhole configurations. More specifically, for positive cosmological constant we have wormholes which expand forever and, for negative cosmological constant we have wormholes which expand to a maximum value and then recolapse. At spatial infinity the energy density and the pressures of the anisotropic phantom matter threading the wormholes vanish; thus these evolving wormholes are asymptotically vacuum $\Lambda$-Friedmann models with either open or closed or flat topologies.

PACS numbers: 04.20.Jb, 04.70.Dy,11.10.Kk
\end{abstract}

\section{INTRODUCTION}

It is well known that in 1917 Einstein added a constant term into his field equations of general relativity allowing static cosmological models for the Universe. This term, called cosmological constant, would create a repulsive gravitational force that does not depend on position nor on time. Later, after Hubble discovered that the universe is expanding, Einstein discarded this constant term, as the "biggest blunder of his life".

However, recent observations are indicating that a cosmological constant may play an essential role in the cosmic expansion of the Universe [1]. According to the standard cosmology the total energy density of the Universe is dominated today by both dark matter and dark energy densities. The dark energy component in general is considered as a kind of vacuum energy homogeneously distributed with negative pressure. The observational data provide compelling evidence for the existence of dark energy which dominates the present day Universe and ac-

\footnotetext{
*mcataldo@ubiobio.cl

†sdelcamp@ucv.cl

${ }^{\ddagger}$ pminning@udec.cl

§pasalgad@udec.cl
}

celerates its expansion.

In principle, any matter content which violates the strong energy condition and possesses a positive energy density and a negative pressure, may cause the dark energy effect of repulsive gravitation. So the main problem of modern cosmology is to identify this form of dark energy that dominates the universe today. Dark energy composed of just a cosmological term $\Lambda$ is fully consistent with existing observational data. A cosmological constant can be associated with a time independent dark energy density. Another candidate is the phantom matter, whose energy density increases with the expansion of the universe [2].

In general relativity one can also consider other gravitational configurations where a cosmological constant, and even the phantom matter, may play an important role. For example the presence of the cosmological constant allows charged and non-charged black hole geometries in $2+1$ gravity [3]. On the other hand wormholes, as well as black holes, are an extraordinary consequence of Einstein's equations of general relativity and, during recent decades, there has been a considerable interest in the field of wormhole physics. For instance, Lorentzian wormhole configurations [4, ,5] may be supported by phantom matter [7, 8, 9] or by a cosmological constant [10, 11, 12]. 
Two separate directions emerged: one relating to static Lorentzian wormholes and the other concerned with time-dependent Lorentzian ones [13, 14, 15]. In both cases the interest has been focused on traversable wormholes, which have no horizons, allowing two-way passage through them. However, most of the efforts are directed to study static configurations which are traversable. The most striking property of such a wormhole is the violation of energy conditions. This implies that the matter supporting the traversable wormholes is exotic [4, 5], which means that it has very strong negative pressures, or even that the energy density is negative, as seen by static observers.

One interesting aspect that we shall consider here is the possibility of sustaining traversable wormhole spacetimes via exotic matter made out of phantom energy. The latter is considered to be a possible candidate for explaining the late time accelerated expansion of the Universe [16]. This phantom energy has a very strong negative pressure and violates the null energy condition, so becoming a most promising ingredient to sustain traversable wormholes. Notice however that in this case we shall use the notion of phantom energy in a more extended sense since, strictly speaking, the phantom matter is a homogeneously distributed fluid, and here it will be an inhomogeneous and anisotropic fluid [6, 7, 9]. It is interesting to note that in Ref. [17] is considered the possibility that the enormous negative pressure in the center of a dark energy star may, in principle, imply a topology change, consequently opening up a tunnel and converting the dark energy star into a wormhole. It should be mentioned that the stability of some specific wormhole static configurations also has been considered [18].

The theoretical construction of wormholes is usually performed by using the method where, in order to have a desired metric, one is free to fix the form of the metric functions, such as the redshift and shape functions, or even the scale factor for evolving wormholes. In this way one may have a redshift function without horizons, or with a desired asymptotic. Unfortunately, in this case we can obtain expressions for the energy and pressure densities which are physically unreasonable.

For constructing wormholes another method can be used. One may impose conditions on the stress-energy tensor threading the wormhole configuration. For example, one can impose the so-called selfdual condition $\rho=\rho_{t}=0$, on the matter supporting the wormhole, where $\rho=T_{\alpha \beta} u^{\alpha} u^{\beta}$ and $\rho_{t}=\left(T_{\alpha \beta}-\frac{1}{2} T g_{\alpha \beta}\right) u^{\alpha} u^{\beta}$ are the energy density measured by a static observer [19]. One can also prescribe the matter content by specifying the equations of state of the radial and of the tangential pressures or by choosing a more specific matter field, such as, for example, a classical scalar field. Notice that, as a simple model of phantom matter, one may consider a classical scalar field with a negative kinetic energy term (the so called ghost or phantom scalar field). Static [20] and non-static wormholes 21] were constructed with the help of such a ghost scalar field. In all these here de- scribed cases one can solve the Einstein field equations in order to find all the metric functions.

In this paper we shall consider the radial and tangential pressures of the matter threading the wormhole to obey barotropic equations of state with constant state parameters coupled to a cosmological constant. More specifically, we shall find all evolving wormhole geometries which have their radial and tangential pressures proportional to the energy density in the presence of a cosmological constant.

The outline of the present paper is as follows: In Sec. II, we briefly review some important aspects of evolving wormholes and write the Einstein equations for matter configurations considered here. In Sec. III, we obtain the general metrics which describe evolving wormholes with anisotropic pressures obeying barotropic equations of state with constant state parameters in the presence of a cosmological constant. In Sec. IV, the properties of the obtained wormhole geometries are studied and discussed.

\section{EVOLVING LORENTZIAN WORMHOLES AND THE EINSTEIN FIELD EQUATIONS}

The metric ansatz of Morris and Thorne [4] for the spacetime which describes a static Lorentzian wormhole is given by

$$
d s^{2}=-e^{2 \Phi(r)} d t^{2}+\frac{d r^{2}}{1-\frac{b(r)}{r}}+r^{2}\left(d \theta^{2}+\sin ^{2} \theta d \varphi^{2}\right),
$$

where $\Phi(r)$ is the redshift function, and $b(r)$ is the shape function since it controls the shape of the wormhole. In order to have a wormhole the functions $b(r)$ and $\Phi(r)$ must satisfy the general constraints discussed by Morris and Thorne in Ref. [4]. These constraints provide a minimum set of conditions which lead, through an analysis of the embedding of the spacelike slice of (1) in a Euclidean space, to a geometry featuring two asymptotically flat regions connected by a bridge. Although asymptotically flat wormhole geometries have been extensively considered in the literature, asymptotically anti-de Sitter wormholes are also of particular interest [22].

Now, the evolving wormhole spacetime may be obtained by a simple generalization of the original Morris and Thorne metric (11) to a time-dependent metric given by

$$
\begin{array}{r}
d s^{2}=-e^{2 \Phi(t, r)} d t^{2}+ \\
a(t)^{2}\left(\frac{d r^{2}}{1-\frac{b(r)}{r}}+r^{2}\left(d \theta^{2}+\sin ^{2} \theta d \varphi^{2}\right)\right),
\end{array}
$$

where $a(t)$ is the scale factor of the universe. Note that the essential characteristics of a wormhole geometry are still encoded in the spacelike section. It is clear that if $b(r) \rightarrow 0$ and $\Phi(t, r) \rightarrow 0$ the (2) metric becomes the flat Friedmann-Robertson-Walker (FRW) metric and, as $a(t) \rightarrow$ const and $\Phi(t, r)=\Phi(r)$ it becomes the static wormhole metric (1). 
Now we shall write the Einstein equations for the (2) metric. In order to simplify the analysis and the physical interpretation we now introduce the proper orthonormal basis as

$$
d s^{2}=-\theta^{(t)} \theta^{(t)}+\theta^{(r)} \theta^{(r)}+\theta^{(\theta)} \theta^{(\theta)}+\theta^{(\varphi)} \theta^{(\varphi)},
$$

where the basis one-forms $\theta^{(\alpha)}$ are given by

$$
\begin{gathered}
\theta^{(t)}=e^{\Phi(t, r)} d t ; \quad \theta^{(r)}=\frac{a(t) d r}{\sqrt{1-\frac{b(r)}{r}}} \\
\theta^{(\theta)}=a(t) r d \theta ; \quad \theta^{(\varphi)}=a(t) r \sin \theta d \varphi .
\end{gathered}
$$

In general, for the metric (2) one might introduce a matter source described by an imperfect fluid, with its four-velocity $u^{(\alpha)}$ oriented along the tetrad $\theta^{(t)}$. Thus for the space-time (2) one might consider a stress-energy tensor of an imperfect fluid containing the coefficients of bulk viscosity, shear viscosity and heat conduction (for the considered metric we might have energy flux in the radial direction), where all of these transport coefficients might be functions of $t$ and/or of $r$. Of course in this case the energy-momentum tensor has also non-diagonal entries. However, as we have pointed out above, we shall use the notion of phantom energy in a slightly more extended sense: We shall consider this traditionally homogeneous and isotropic exotic source to be generalized to an inhomogeneous and anisotropic fluid, but still with a diagonal energy-momentum tensor (as is usually considered in phantom cosmologies). This means that, for the tetrad basis (4), the only nonzero components of the energy-momentum tensor $T_{(\mu)(\nu)}$ are the diagonal terms $T_{(t)(t)}, T_{(r)(r)}, T_{(\theta)(\theta)}$ and $T_{(\varphi)(\varphi)}$, which are given by

$$
\begin{array}{r}
T_{(t)(t)}=\rho(t, r), T_{(r)(r)}=p_{r}(t, r)=-\tau(t, r), \\
T_{(\theta)(\theta)}=T_{(\varphi)(\varphi)}=p_{l}(t, r),
\end{array}
$$

where the quantities $\rho(t, r), p_{r}(t, r), \tau(t, r)\left(=-p_{r}(t, r)\right)$, and $p_{l}(t, r)\left(=p_{\varphi}(t, r)=p_{\theta}(t, r)\right)$ are respectively the energy density, the radial pressure, the radial tension per unit area, and the lateral pressure as measured by observers who always remain at rest at constant $r, \theta, \varphi$. Thus for the evolving spherically symmetric wormhole metric (2) the Einstein equations with cosmological constant $\Lambda$

$$
R_{(\alpha)(\beta)}-\frac{R}{2} g_{(\alpha)(\beta)}=-\kappa T_{(\alpha)(\beta)}-\Lambda g_{(\alpha)(\beta)}
$$

are given by

$$
\begin{array}{r}
3 e^{-2 \phi(t, r)} H^{2}+\frac{b^{\prime}}{a^{2} r^{2}}=\kappa \rho(t, r)+\Lambda, \\
-e^{-2 \phi(t, r)}\left(2 \frac{\ddot{a}}{a}+H^{2}\right)-\frac{b}{a^{2} r^{3}}+2 e^{-2 \phi(t, r)} H \frac{\partial \phi}{\partial t}+ \\
\frac{2}{r^{2} a^{2}}(r-b) \frac{\partial \phi}{\partial r}=\kappa p_{r}(t, r)-\Lambda, \\
-e^{-\phi(t, r)}\left(2 \frac{\ddot{a}}{a}+H^{2}\right)+\frac{b-r b^{\prime}}{2 a^{2} r^{3}}+
\end{array}
$$

$$
\begin{array}{r}
2 e^{-\phi(t, r)} H \frac{\partial \phi}{\partial t}+\frac{1}{2 a^{2} r^{2}}\left(2 r-b-r b^{\prime}\right) \frac{\partial \phi}{\partial r}+ \\
\frac{1}{a^{2} r}(r-b)\left(\left(\frac{\partial \phi}{\partial r}\right)^{2}+\frac{\partial^{2} \phi}{\partial r^{2}}\right)=\kappa p_{l}(t, r)-\Lambda, \\
2 e^{-\phi(t, r)} \sqrt{\frac{r-b(r)}{r}} \frac{\partial \phi}{\partial r} \dot{a}=0,
\end{array}
$$

where $\kappa=8 \pi G, H=\dot{a} / a$, and an overdot and a prime denote differentiation $d / d t$ and $d / d r$ respectively.

It is direct to see that, for the diagonal energymomentum tensor (5), Eq. (9) gives some constraints on relevant metric functions which separate the wormhole solutions into two branches: one static branch given by the condition $\dot{a}=0$ and another non-static branch for $\partial \phi / \partial r=0$. This latter condition implies that the redshift function can only be a function of $t$, i.e. $\phi(t, r)=f(t)$ so, without any loss of generality, by rescaling the time coordinate we can set $\phi(t, r)=0$.

It is interesting to note that the static branch with $\phi(r) \neq$ const allows one to have isotropic pressures for the matter threading the wormhole (see for example 7$]$ ). As we shall see below, since for the non-static branch we must fulfill the condition $\phi(t, r)=0$, the matter pressures cannot be isotropic at all.

In what follows we shall restrict our discussion to evolving wormhole geometries, so we must consider the nonstatic branch. Thus, as we stated above, without any loss of generality we shall require $\phi(t, r)=0$. By using the conservation equation $T_{\nu ; \mu}^{\mu}=0$, we have that

$$
\begin{gathered}
\frac{\partial \rho}{\partial t}+H\left(3 \rho+p_{r}+2 p_{l}\right)=0, \\
\frac{2\left(p_{l}-p_{r}\right)}{r}=\frac{2\left(p_{l}+\tau\right)}{r}=\frac{\partial p_{r}}{\partial r} .
\end{gathered}
$$

From these equations we see that for an isotropic pressure, i.e. $p_{l}=p_{r}=p$, we have to require $\partial p_{r} / \partial r=0$, so the pressure will depend only on time $t$, obtaining the standard cosmological conservation equation $\dot{\rho}+3 H(\rho+$ $p)=0$. If we want to study wormholes with pressures depending on both variables $t$ and $r$, we must consider only anisotropic pressures, thus requiring $p_{r} \neq p_{l}$.

In the following we are interested in studying matter sources threading the wormhole described by barotropic equations of state with constant state parameters. Thus we shall require that

$$
\begin{array}{r}
\tau(t, r)=-p_{r}(t, r)=-\omega_{r} \rho(t, r), \\
p_{l}(t, r)=\omega_{l} \rho(t, r),
\end{array}
$$

where $\omega_{r}$ and $\omega_{l}$ are constant state parameters. Clearly, the requirement (12) with $\omega_{r}=\omega_{l}=\omega$ allows us to connect the evolving wormhole spacetime (2) with the standard FRW cosmologies, where the isotropic pressure density is expressed as $p=\omega \rho$, with constant state parameter $\omega$.

Now, with the help of the conservation equations (10) and (11) we can easily solve the Einstein equations (6) 
(9). Note that in these equations the cosmological constant is not present, so we can solve the field equations following the procedure of Ref. [9]. From the structure of these conservation equations we see that one can write the energy density in the form $\rho(t, r)=\rho_{t}(t) \rho_{r}(r)$. Thus from the conservation equations (10) and (11) we obtain

$$
\begin{gathered}
\rho_{t}(t)=C_{1} a^{-\left(3+\omega_{r}+2 \omega_{l}\right)}, \\
\rho_{r}(r)=C_{2} r^{2\left(\omega_{l}-\omega_{r}\right) / \omega_{r}},
\end{gathered}
$$

where $C_{1}$ and $C_{2}$ are integration constants, obtaining for the full energy density

$$
\rho(t, r)=C r^{2\left(\omega_{l}-\omega_{r}\right) / \omega_{r}} a^{-\left(3+\omega_{r}+2 \omega_{l}\right)},
$$

where we have introduced a new constant $C$ in order to redefine $C_{1}$ and $C_{2}$.

Now, by subtracting Eqs. (77) and (8), and using the full energy density (15), we obtain the differential equation

$$
\frac{\kappa\left(\omega_{l}-\omega_{r}\right) C r^{2\left(\omega_{l}-\omega_{r}\right) / \omega_{r}}}{a^{\left(3+\omega_{r}+2 \omega_{l}\right)}}=\frac{3 b-r b^{\prime}}{2 a^{2} r^{3}} .
$$

It is straightforward to see that in order to have a solution for the shape function $b=b(r)$ we must impose the constraint

$$
\omega_{r}+2 \omega_{l}+1=0
$$

on the state parameters $\omega_{r}$ and $\omega_{l}$, thus obtaining for the shape function

$$
b(r)=C_{3} r^{3}-\kappa C \omega_{r} r^{-1 / \omega_{r}},
$$

where $C_{3}$ is a new integration constant. Notice that the constraint (17) implies that the radial and tangential pressures are given by

$$
p_{r}=\omega_{r} \rho, \quad p_{l}=-\frac{1}{2}\left(1+\omega_{r}\right) \rho,
$$

so the energy density and pressures satisfy the following relation:

$$
\rho+p_{r}+2 p_{l}=0 .
$$

Now, from Eqs. (6), (15), (18) and taking into account the constraint (17) we obtain the following master equation for the scale factor:

$$
3 H^{2}=-\frac{3 C_{3}}{a^{2}}+\Lambda \text {. }
$$

In the following paragraph, with the help of this equation, we shall determine the scale factor, and then the specific form of the energy density (15).

Lastly, note that there is another branch of spherically symmetric solutions to Eqs. (6)-(8). By adding these equations and taking into account Eqs. (12) and (15) we obtain the equation

$6 \frac{\ddot{a}}{a}-2 \Lambda=-\kappa\left(1+\omega_{r}+2 \omega_{l}\right) C r^{2\left(\omega_{l}-\omega_{r}\right) / \omega_{r}} a^{-\left(3+\omega_{r}+2 \omega_{l}\right)}$.
Now, if we take $\omega_{r}=\omega_{l}=\omega$, we obtain a differential equation for $a(t)$ and $\Lambda$ which has a first integral given by

$$
3\left(\frac{\dot{a}}{a}\right)^{2}+\frac{\text { Const }}{a^{2}}=\kappa C a(t)^{-3(1+\omega)}+\Lambda .
$$

This differential equation is the standard Friedmann equation for a FRW universe filled with an ideal fluid (with $p(t)=\omega \rho(t)$ ) and a cosmological constant. The term Const $/ a^{2}$ is related to the curvature term while the energy density scales as $\rho \sim a^{-3(1+\omega)}$.

It is worth noticing that, if we take $1+\omega_{r}+2 \omega_{l}=0$ (see Eq. (17)), we obtain from Eq. (22) the differential equation $3 \ddot{a} / a=\Lambda$ which has a first integral of the form of the master equation (21).

\section{WORMHOLE SOLUTIONS}

Now we shall study the specific wormhole configurations which should be allowed by the master equation (21). The case $\Lambda=0$ (which implies that $a(t)=$ $\sqrt{-C_{3}} t+$ Const) was considered before in Ref. [9] so we shall restrict ourselves here to the case $\Lambda \neq 0$. To start with, we shall consider first the static case of a de Sitter wormhole.

\section{A. The $C_{3}=0$ case: The de Sitter wormhole}

First, let us consider the case $C_{3}=0$ in Eq. (21). In this case the solution is given by

$$
a(t)=a_{0} e^{ \pm \sqrt{\Lambda / 3} t}
$$

and is allowed only for $\Lambda>0$. Thus the metric (2) takes the form

$$
\begin{array}{r}
d s^{2}=d t^{2}+a_{0}^{2} e^{2 \pm \sqrt{\Lambda / 3} t} \times \\
\left(\frac{d r^{2}}{1+\kappa C \omega_{r} r^{-\left(1+1 / \omega_{r}\right)}}+r^{2}\left(d \theta^{2}+\sin ^{2} \theta d \varphi^{2}\right)\right)
\end{array}
$$

describing contracting and expanding wormholes. If we choose the plus sign in Eq. (24) we obtain an inflationary scale factor, which gives an exponential expansion for an inflating wormhole. This kind of wormholes was considered before by Roman in Ref. [11]; however, in this work the resulting properties are associated with a stressenergy tensor of a general form, without imposing a specific equation of state among the energy density and the pressures. In this case, for an inflating wormhole, we obtain from Eq. (12) that the anisotropic pressures take the form (19) where the energy density is given by

$$
\rho(t, r) \sim \frac{r^{-\left(1+3 \omega_{r}\right) / \omega_{r}}}{e^{2 \sqrt{\Lambda / 3} t}} .
$$


Since we want to study a wormhole configuration sustained via exotic matter made out of phantom energy, we are interested in the range $\omega_{r}<-1$ for the main state parameter of the solution. Clearly, in this case for $r \rightarrow \infty$ we have $\rho \rightarrow 0$, thus at spatial infinity this wormhole looks like a de Sitter universe.

Now we shall rewrite this wormhole solution in a more appropriate form. From the condition for the throat that the $r$-coordinate has a minimum at $r_{0}$, i.e. $g_{r r}^{-1}\left(r_{0}\right)=0$, we obtain for the integration constant $C=-\frac{r_{0}^{\left(1+\omega_{r}\right) / \omega_{r}}}{\kappa \omega_{r}}$, yielding for the shape function and the energy density

$$
b(r)=r_{0}\left(\frac{r}{r_{0}}\right)^{-1 / \omega_{r}}, \kappa \rho(t, r)=-\frac{\left(r / r_{0}\right)^{-\left(1+3 \omega_{r}\right) / \omega_{r}}}{\omega_{r} r_{0}^{2} e^{2 \sqrt{\Lambda / 3} t}},
$$

respectively. Thus the metric (25) takes the form

$$
\begin{array}{r}
d s^{2}=d t^{2}+a_{0}^{2} e^{2 \pm \sqrt{\Lambda / 3} t} \times \\
\left(\frac{d r^{2}}{1-\left(r / r_{0}\right)^{-\left(1+\omega_{r}\right) / \omega_{r}}}+r^{2}\left(d \theta^{2}+\sin ^{2} \theta d \varphi^{2}\right)\right) .
\end{array}
$$

The radial coordinate $r$ has a range that increases from a minimum value at $r_{0}$, corresponding to the wormhole throat, to infinity. From Eqs. (19), (27) and (28) we can see that for $\omega_{r}<-1$ the wormhole solution is an asymptotically de Sitter universe with a matter content having a radial pressure with a phantom equation of state and everywhere positive energy density. Note that for $\omega_{r}>0$ we also have a wormhole solution with an asymptotically de Sitter universe, but in this case the energy density is negative everywhere. For $\Lambda=0$ we have a static wormhole solution considered before in Ref. 7] (see also Ref. [9]).

\section{B. The $C_{3} \neq 0$ case}

Let us now explore the features of the general solution for the above found evolving Lorentzian wormholes supported by the considered anisotropic matter and cosmological constant. From the master equation (21) we can see that $H^{2}>0$ only for the following combinations of the parameters $C_{3}$ and $\Lambda: C_{3}>0$ and $\Lambda>0$, or $C_{3}<0$ and $\Lambda>0$, or $C_{3}<0$ and $\Lambda<0$. The general solution for Eq. (21) with $\Lambda \neq 0$ is given by

$$
a(t)=\frac{\sqrt{3}}{6 \sqrt{\Lambda}}\left[\frac{9 C_{3}+C_{4}^{2} e^{ \pm 2 \sqrt{\Lambda / 3} t}}{C_{4} e^{ \pm \sqrt{\Lambda / 3} t}}\right],
$$

where $C_{4}$ is a new integration constant. For the cases enumerated before, the solution may be rewritten in the form shown in table [1 where $\phi_{0}$ is a constant.

Thus the solution for $C_{3} \neq 0$ is given by the metric (2) with $\Phi(t, r)=0$, the shape function (18) and the scale factors indicated in Table [ However, it must be noted that in this solution the radial coordinate may be rescaled

\begin{tabular}{|c|c|c|}
\hline$a_{\Lambda}(t)$ & $C_{3} \neq 0$ & $\Lambda \neq 0$ \\
\hline$\sqrt{\frac{3 C_{3}}{\Lambda}} \cosh \left(\sqrt{\frac{\Lambda}{3}} t+\phi_{0}\right)$ & $>0$ & $>0$ \\
\hline$\sqrt{\frac{-3 C_{3}}{\Lambda}} \sinh \left(\sqrt{\frac{\Lambda}{3}} t+\phi_{0}\right)$ & $<0$ & $>0$ \\
\hline$\sqrt{\frac{3 C_{3}}{\Lambda}} \sin \left(\sqrt{\frac{-\Lambda}{3}} t+\phi_{0}\right)$ & $<0$ & $<0$ \\
\hline
\end{tabular}

TABLE I: The table shows the possible scale factors derived from Eq. (21) and corresponding to the cases $C_{3} \neq 0$ and $\Lambda \neq 0$.

in order to absorb the integration constant $C_{3}$. In this case we obtain the final metric given by

$$
\begin{array}{r}
d s^{2}=-d t^{2}+a_{\Lambda}^{2}(t) \times \\
\left(\frac{d r^{2}}{1-k r^{2}+\kappa C \omega_{r} r^{-\left(1+\omega_{r}\right) / \omega_{r}}}+r^{2}\left(d \theta^{2}+\sin ^{2} \theta d \varphi^{2}\right)\right),
\end{array}
$$

where $k=1$ for $C_{3}>0, k=-1$ for $C_{3}<0$. Note also that we can include in the rescaled metric (30) the case considered in the previous subsection making $k=0$ for $C_{3}=0$. We summarize in Table $\Pi$ all possible scale factors for the found wormhole solutions (30).

\begin{tabular}{|c|c|c|}
\hline$a_{\Lambda}(t)$ & $k$ & $\Lambda$ \\
\hline const & 0 & 0 \\
\hline$t+$ const & -1 & 0 \\
\hline$a_{0} e^{ \pm \sqrt{\Lambda / 3}} t$ & 0 & $>0$ \\
\hline$\sqrt{\frac{3}{\Lambda}} \cosh \left(\sqrt{\frac{\Lambda}{3}} t+\phi_{0}\right)$ & 1 & $>0$ \\
\hline$\sqrt{\frac{3}{\Lambda}} \sinh \left(\sqrt{\frac{\Lambda}{3}} t+\phi_{0}\right)$ & -1 & $>0$ \\
\hline$\sqrt{\frac{-3}{\Lambda}} \sin \left(\sqrt{\frac{-\Lambda}{3}} t+\phi_{0}\right)$ & -1 & $<0$ \\
\hline
\end{tabular}

TABLE II: The table shows all the possible scale factors for the general solution (30) of an evolving Lorentzian wormhole with the radial tension and the tangential pressure having barotropic equations of state (12) with constant state parameters. Here we have included the case $k=-1, \Lambda=0$ considered before in Ref. [9].

In this case evolving wormholes have a matter source with the anisotropic pressures given by Eq. (19) where now the energy density has the form

$$
\rho(t, r)=\frac{C r^{-\left(1+3 \omega_{r}\right) / \omega_{r}}}{a_{\Lambda}^{2}(t)},
$$

with $a_{\Lambda}$ given in Table II

Now we shall rewrite the wormhole metric (30) in a more appropriate form. From the condition $g_{r r}^{-1}(r=$ $\left.r_{0}\right)=0$, we obtain for the integration constant

$$
C=\frac{\left(k r_{0}^{2}-1\right)}{\kappa \omega_{r}} r_{0}^{\left(1+\omega_{r}\right) / \omega_{r}}
$$

yielding for the shape function and the metric component 
$g_{r r}$

$$
\begin{gathered}
b(r)=r_{0}\left(\frac{r}{r_{0}}\right)^{-1 / \omega_{r}} \\
+k r_{0}^{3}\left(\frac{r}{r_{0}}\right)^{3}\left(1-\left(\frac{r}{r_{0}}\right)^{-\left(1+3 \omega_{r}\right) / \omega_{r}}\right), \\
a_{\Lambda}^{2}(t) g_{r r}^{-1}=1-\left(\frac{r}{r_{0}}\right)^{-\left(1+\omega_{r}\right) / \omega_{r}} \\
-k r_{0}^{2}\left(\frac{r}{r_{0}}\right)^{2}\left(1-\left(\frac{r}{r_{0}}\right)^{-\left(1+3 \omega_{r}\right) / \omega_{r}}\right),
\end{gathered}
$$

respectively. This implies that the wormhole throat is located at $r_{0}$. In this case the energy density of the matter threading the wormhole takes the following form:

$$
\kappa \rho(t, r)=\frac{k r_{0}^{2}-1}{r_{0}^{2} \omega_{r} a_{\Lambda}(t)^{2}}\left(\frac{r}{r_{0}}\right)^{-\left(1+3 \omega_{r}\right) / \omega_{r}} .
$$

Clearly, in order to have an evolving wormhole we must require $\omega_{r}<-1$ or $\omega_{r}>0$ (in both of these cases, in the $g_{r r}$ metric component, $\left(1+\omega_{r}\right) / \omega_{r}>0$ and $\left.\left(1+3 \omega_{r}\right) / \omega_{r}>0\right)$, implying that the phantom energy can support the existence of evolving wormholes in the presence of a cosmological constant. As before, for a matter made of phantom energy with $\omega_{r}<-1$, we have that at spatial infinity its energy density vanishes. Thus the wormhole configurations with $k=-1,1$ (or equivalently $C_{3} \neq 0$ ) are asymptotically vacuum $\Lambda$-Friedmann models with open and closed topologies respectively with the corresponding scale factors of the table II. For $\omega_{r}>0$ we have the same behavior.

\section{PROPERTIES OF THE SOLUTIONS AND DISCUSSION}

Now we shall consider some characteristic properties of the above found evolving wormhole geometries. Essentially, as we shall see, these wormholes coupled to a cosmological constant have the same properties as have the evolving wormholes with $\Lambda=0$ discussed in Ref. [9], since the cosmological constant directly controls the behavior of the scale factor $a(t)$ and not the shape function $b(r)$ of the spacelike section of the metric (2). However it must be noticed that in this case we have a richer variety of topologies since now we can consider evolving wormhole configurations with $k=0$ and $k=1$, while only the case $k=-1$ for $\Lambda=0$. Effectively, for example, from the metric (30) we can see that for wormholes supported by phantom matter at spatial infinity $(r \rightarrow \infty)$ we have the following asymptotic metric:

$$
\begin{array}{r}
d s^{2} \approx-d t^{2}+ \\
a_{\Lambda}^{2}(t)\left(\frac{d r^{2}}{1-k r^{2}}+r^{2}\left(d \theta^{2}+\sin ^{2} \theta d \varphi^{2}\right)\right) .
\end{array}
$$

This metric has slices $t=$ const which are 3 -spaces of constant curvature: open for $k=-1$, flat for $k=0$ and closed for $k=1$. This implies that the asymptotic metric (35) is foliated with spaces of constant curvature.

Now the presence of the cosmological constant of course indicates that these wormholes are not asymptotically flat. If we calculate the Riemann tensor for the metric (30), we find that its independent non-vanishing components are

$$
\begin{array}{r}
R_{(\theta)(r)(r)(\theta)}=R_{(\varphi)(r)(r)(\varphi)}= \\
\frac{\dot{a}^{2}+k+\frac{1}{2} \kappa C\left(1+\omega_{r}\right) r^{-\left(1+3 \omega_{r}\right) / \omega_{r}}}{a^{2}}, \\
R_{(\varphi)(\theta)(\theta)(\varphi)}=\frac{\dot{a}^{2}+k-\frac{1}{2} \kappa C \omega_{r} r^{-\left(1+3 \omega_{r}\right) / \omega_{r}}}{a^{2}}, \\
R_{(t)(\theta)(t)(\theta)}=R_{(t)(\varphi)(t)(\varphi)}=R_{(t)(r)(t)(r)}=\frac{\ddot{a}}{a} .
\end{array}
$$

From these expressions and the scale factors given in Table III we see that at spatial infinity, i.e. $r \rightarrow \infty$, these Riemann tensor components do not vanish for a wormhole with $\omega_{r}<-1$ or $\omega_{r}>0$, except for the case $\Lambda=0$ as stated in Ref. [9]. In conclusion, regardless of the energy density (31) vanishing for $r \rightarrow \infty$, the Riemann tensor components do not vanish, due to the presence of the cosmological constant $\Lambda$.

Let us now consider the energy conditions. It is well known that, in all cases, the violation of the weak energy condition (WEC) is a necessary condition for a static wormhole to exist. In general, for the energy-momentum tensor (5), WEC reduces to the following inequalities:

$$
\begin{array}{r}
\rho(t, r) \geq 0, \quad \rho(t, r)+p_{r}(t, r) \geq 0, \\
\rho(t, r)+p_{l}(t, r) \geq 0,
\end{array}
$$

which may be rewritten by using the expressions (12) and (17) as follows:

$$
\begin{aligned}
\rho(t, r) \geq 0, \quad\left(1+\omega_{r}\right) \rho(t, r) \geq 0, \\
\left(1-\omega_{r}\right) \rho(t, r) \geq 0 .
\end{aligned}
$$

Thus, for the found gravitational configurations (for which we must require $\omega_{r}<-1$ or $\omega_{r}>0$ in order to have a wormhole), Eqs. (34) and (38) imply that for:

- $k=0$ or $k=-1$ and $\rho>0$, we must require $\omega_{r}<-1$, so the WEC is always violated. Note that this is independent of the value of the cosmological constant.

- $k=1$ (in this case $\Lambda>0$ ) and $\rho>0$, we may require $\omega_{r}<-1$ for $r_{0}^{2}<1$ (and the WEC is always violated) or require $0<\omega_{r}<1$ for $r_{0}^{2}>1$ (and the violation of WEC is avoided). Unfortunately this latter case must be ruled out for the consideration of evolving wormhole configurations as we shall see below. 
Note that for the case $k=1$ the value $r_{0}=1$ implies that $\rho=0$ and $b(r)=r^{3}$, so we have in this case a homogeneous and isotropic closed $\Lambda$-FRW cosmology.

In order to dilucidate whether or not the shape of the wormhole is maintained, we must check the fulfillment of the flaring out condition, which is given by

$$
\frac{d^{2} \bar{r}}{d \bar{z}^{2}}=\frac{\bar{b}-\bar{b}^{\prime} r}{2 \bar{b}^{2}}=\frac{b-b^{\prime} r}{2 a\left(t_{0}\right) b^{2}}>0,
$$

where $\bar{r}=a_{\Lambda}\left(t_{0}\right) r, \bar{b}(\bar{r})=a_{\Lambda}\left(t_{0}\right) b(r)$ (for a detailed discussion see Ref. [9]). Taking into account the shape function $b(r)$ of Eq. (33) we obtain

$$
\begin{gathered}
\frac{d^{2} \bar{r}}{d \bar{z}^{2}}=-\left(\frac{r}{r_{0}}\right)^{1 / \omega_{r}} \times \\
\frac{2 D \omega_{r} r^{3}\left(r / r_{0}\right)^{1 / \omega_{r}}+\left(1+\omega_{r}\right) r_{0}\left(k r_{0}^{2}-1\right)}{2 \omega_{r}\left(k r^{3}\left(r / r_{0}\right)^{1 / \omega_{r}}+r_{0}\left(1-k r_{0}^{2}\right)\right)^{2}} .
\end{gathered}
$$

Clearly if $k=0,-1$ this constraint is satisfied for the entire range of the radial coordinate $r$. If $k=1$ this constraint is always violated for $r_{0}>1$, and for $r_{0}<1$ it may be satisfied. Thus we can have wormholes with $k=1$ only for $r_{0}<1$ and then WEC is violated.

On the other hand, from Eq. (22) and the constraint (17) we conclude that the expansion of the wormhole is accelerated due to the presence of the cosmological constant. So this family of evolving wormholes, supported by an anisotropic phantom energy, may expand with acceleration for $k=1,0,-1$. More specifically, from the form of the scale factors of Table $\amalg$, we conclude that for the cases with $\Lambda>0$ the wormhole expands forever, and for $\Lambda<0$ the wormhole expands to a maximum value and then recolapses.

The shape of a wormhole is determined by $b(r)$ as viewed, for example, in an embedding diagram in a flat 3 -dimensional Euclidean space $R^{3}$. To construct in our case such a diagram of a wormhole we can follow the procedure described in Ref. [9]. In order to have a good embedding we must impose the condition $b(r) \geq 0$. Clearly from Eq. (33) we see that for $k=0$ and $k=1$ the shape function $b(r)$ is positive for all $r>r_{0}$. For $k=-1$ the shape function is positive for $r_{0}<r<r_{\max }$ where $r_{\max }=r_{0}\left(1+\frac{1}{r_{0}^{2}}\right)^{\omega_{r} /\left(1+3 \omega_{r}\right)}$.

Let us now consider the tidal forces experienced by a traveller while crossing this kind of wormholes. We recall that the tidal acceleration must not exceed one Earth gravity, i.e. $g_{\oplus}=9.8 \mathrm{~m} / \mathrm{s}^{2}$, in order for wormhole travel to be at all convenient for human beings [4]. The calculus of these tidal forces may be simplified by introducing a new orthonormal reference frame which moves at a constant speed $v$ with respect to observers who always remain at rest at constant $r, \theta$ and $\varphi$ (see Ref. 9] and also cites therein).

Thus, for the generic metric (2) (with $\Phi(t, r)=0$ ), and by considering the size of the traveller's body (i.e. head to feet) to be $\sim 2(\mathrm{~m})$, the Riemann tensor components in this new basis are constrained to be

$$
\left|R_{\hat{1}^{\prime} \hat{0}^{\prime} \hat{1}^{\prime} \hat{0}}\right|=\left|\frac{\ddot{a}}{a}\right| \leq \frac{g_{\oplus}}{c^{2} \times 2 m} \simeq \frac{1}{\left(10^{8} m\right)^{2}},
$$

and

$$
\begin{array}{r}
\left|R_{\hat{2}^{\prime} \hat{0}^{\prime} \hat{2}^{\prime} \hat{0}^{\prime}}\right|=\left|R_{\hat{3}^{\prime} \hat{0}^{\prime} \hat{3}^{\prime} \hat{0}^{\prime}}\right|= \\
\left|\gamma^{2} \frac{\ddot{a}}{a}-\frac{\gamma^{2} \beta^{2}}{2 a^{2} r^{3}}\left(2 \dot{a}^{2} r^{3}-b+r b^{\prime}\right)\right| \leq \\
\frac{g_{\oplus}}{c^{2} \times 2 m} \simeq \frac{1}{\left(10^{8} m\right)^{2}},
\end{array}
$$

where $\gamma=1 / \sqrt{1-\beta^{2}}$ with $\beta=v / c$.

In this case the radial tidal constraint (41) can be regarded as directly constraining the acceleration of the expansion of the wormhole, while the lateral tidal constraint (42) can be regarded as constraining the speed $v$ of the traveller while crossing the wormhole.

In particular, the evolving wormholes considered in this paper evolve with scale factors shown in Table II. This implies that the expansion is not accelerated (i.e. $\ddot{a}=0$ ) only for the cases $\Lambda=0$, thus satisfying the constraint (41). Now, by taking into account Eq. (22), we conclude that for all $\Lambda \neq 0$ cases of Table II, the radial tidal constraint (41) implies the following constraint on the cosmological constant:

$$
\left|\frac{\Lambda}{3}\right| \leq \frac{g_{\oplus}}{c^{2} \times 2 m} \simeq \frac{1}{\left(10^{8} m\right)^{2}} .
$$

Note that this constraint on the cosmological constant is valid for all values of $k=-1,0,1$.

Now, by using the Einstein equation (8) with Eqs. (17) and (22), the lateral tidal constraint (42) may be rewritten as follows:

$$
\left|\frac{\Lambda}{3} \gamma^{4}-\frac{1}{2} \gamma^{2} \beta^{2}\left(1+\omega_{r}\right) \kappa \rho\right| \leq \frac{g_{\oplus}}{c^{2} \times 2 m} \simeq \frac{1}{\left(10^{8} m\right)^{2}} .
$$

Since constraint (41) implies that the cosmological constant $\Lambda \leq 10^{-16} \mathrm{~m}^{-2}$ and the motion of the traveller be non-relativistic (i.e. $v<<c, \gamma \approx 1$ ), the above constraint may be rewritten as

$$
\left|\frac{1}{2}\left(\frac{v}{c}\right)^{2}\left(1+\omega_{r}\right) \kappa \rho\right| \leq \frac{g_{\oplus}}{c^{2} \times 2 m} \simeq \frac{1}{\left(10^{8} m\right)^{2}} .
$$

Thus the lateral tidal constraint (42) can be regarded more exactly as constraining both the speed $v$ of the traveller and the energy density of the matter threading the wormhole. By taking into account the expression for the energy density (34), we may rewrite Eq. (44) as follows:

$$
\left|\frac{v^{2}\left(1+\omega_{r}\right)\left(k r_{0}^{2}-1\right)}{r_{0}^{2} \omega_{r} a_{\Lambda}(t)^{2}}\left(\frac{r}{r_{0}}\right)^{-\left(1+3 \omega_{r}\right) / \omega_{r}}\right| \lesssim g_{\oplus} .
$$


As we can see, this constraint is more severe at the wormhole throat, thus evaluating it at $r=r_{0}$ we obtain

$$
\left|\frac{v^{2}\left(1+\omega_{r}\right)\left(k r_{0}^{2}-1\right)}{r_{0}^{2} \omega_{r} a_{\Lambda}(t)^{2}}\right| \lesssim g_{\oplus}
$$

Thus we conclude that for wormholes with $k=-1,0,1$ and $\Lambda \neq 0$ it is possible to fulfill the constraint (46) for some $t \geq t_{\min }>0$. For example for the case $k=0$ and $a(t)=a_{0} e^{\sqrt{\Lambda / 3} t}$ we have that the constraint (46) is saturated for

$$
t_{e_{0}}=\frac{1}{2 \sqrt{\Lambda / 3}} \ln \left(-\frac{v^{2}\left(1+\omega_{r}\right)}{g_{\oplus} r_{0}^{2} \omega_{r} a_{0}^{2}}\right)
$$

with $-\frac{v^{2}\left(1+\omega_{r}\right)}{g_{\oplus} r_{0}^{2} \omega_{r} a_{0}^{2}}>1$, thus for any $t \geq t_{e_{0}}>0$ the men- tioned lateral tidal constraint will be satisfied.

\section{ACKNOWLEDGEMENTS}

This work was supported by CONICYT through Grants FONDECYT N ${ }^{0} 1080530$ and 1070306 (MC, SdC and PS), and by Dirección de Investigación de la Universidad del Bío-Bío (MC). SdC also was supported by PUCV grant $\mathrm{N}^{0} 123.787 / 2008$ and P.S. and P.M. by Universidad de Concepción through DIUC Grant $\mathrm{N}^{0}$ 208.011.048-1.0.
[1] C. Armendariz-Picon, V. F. Mukhanov and P. J. Steinhardt, Phys. Rev. Lett. 85, 4438 (2000); A. I. Arbab, Class. Quant. Grav. 20, 93 (2003); M. Giovannini, Int. J. Mod. Phys. A 22, 2697 (2007).

[2] E. J. Copeland, M. Sami and S. Tsujikawa, Int. J. Mod. Phys. D 15, 1753 (2006)

[3] M. Banados, C. Teitelboim and J. Zanelli, Phys. Rev. Lett. 69, 1849 (1992); C. Martinez, C. Teitelboim and J. Zanelli, Phys. Rev. D 61, 104013 (2000); M. Cataldo, N. Cruz, S. del Campo and A. Garcia, Phys. Lett. B 484, 154 (2000); M. Cataldo, Phys. Lett. B 529, 143 (2002).

[4] M.S. Morris and K.S. Thorne, Am. J. Phys. 56, 395 (1988); M.S. Morris, K.S. Thorne and U. Yurtsever, Phys. Rev. Lett. 61, 1446 (1988).

[5] M. Visser, Lorentzian Wormholes: From Einstein to Hawking, (AIP, New York, 1995); M. Visser, S. Kar, N. Dadhich Phys. Rev. Lett. 90201102 (2003); N. Dadhich, S. Kar, S. Mukherjee and M. Visser, Phys. Rev. D 65, 064004 (2002).

[6] S. V. Sushkov, Phys. Rev. D 71, 043520 (2005).

[7] F. S. N. Lobo, Phys. Rev. D 71, 084011 (2005).

[8] R. Garattini and F. S. N. Lobo, Class. Quant. Grav. 24, 2401 (2007) ; F. S. N. Lobo, Phys. Rev. D 71, 124022 (2005); O. B. Zaslavskii, Phys. Rev. D 72, 061303 (2005); F. Rahaman, M. Kalam, M. Sarker and K. Gayen, Phys. Lett. B 633, 161 (2006); P. K. F. Kuhfittig, Class. Quant. Grav. 23, 5853 (2006); P. F. Gonzalez-Diaz, Phys. Lett. B 632, 159 (2006).

[9] M. Cataldo, P. Labraña, S. del Campo, J. Crisostomo and P. Salgado, Phys. Rev. D 78, 104006 (2008).

[10] J. P. S. Lemos, F. S. N. Lobo and S. Quinet de Oliveira, Phys. Rev. D 68, 064004 (2003).

[11] T.A. Roman, Phys. Rev. D 47, 1370 (1993).

[12] M. S. R. Delgaty and R. B. Mann, Int. J. Mod. Phys. D 4, 231 (1995); B. N. Esfahani, Gen. Rel. Grav. 37, 271 (2005); S. W. Kim, Phys. Lett. A 166, 13 (1992).

[13] E. Teo, Phys. Rev. D 58, 024014 (1998); V.M. Khatsymovsky, Phys. Lett. B 429, 254 (1998); P. K. F. Kuhfittig, Phys. Rev. D 67, 064015 (2003); Tonatiuh
Matos, D. Nunez Class. Quant. Grav. 23, 4485 (2006); Mubasher Jamil, Muneer Ahmad Rashid, Electromagnetic field around a slowly rotating wormhole arXiv: 0805.0966 [astro-ph].

[14] S. Kar, Phys. Rev. D 49, 862 (1994); S. Kar and D. Sahdev, Phys. Rev. D 53, 722 (1996).

[15] F.S.N. Lobo, Exotic solutions in General Relativity: Traversable wormholes and 'warp drive' spacetimes, ePrint: arXiv:0710.4474 [gr-qc]; A. V. B. Arellano and F. S. N. Lobo; Class. Quant. Grav. 23, 5811 (2006); A. V. B. Arellano and F. S. N. Lobo; Class. Quant. Grav. 23, 7229 (2006).

[16] S. Nojiri, S. D. Odintsov and S. Tsujikawa, Phys. Rev. D 71, 063004 (2005); P. F. Gonzalez-Diaz, Phys. Rev. D 68, 021303 (2003); P. F. Gonzalez-Diaz, Phys. Lett. B 586, 1 (2004); M. Cataldo, N. Cruz and S. Lepe, Phys. Lett. B 619, 5 (2005); G. Izquierdo and D. Pavon, Phys. Lett. B 633, 420 (2006).

[17] A. DeBenedictis, R. Garattini and F. S. N. Lobo, arXiv:0808.0839 [gr-qc].

[18] J. P. S. Lemos and F. S. N. Lobo, Phys. Rev. D 78, 044030 (2008); J. A. Gonzalez, F. S. Guzman and O. Sarbach, arXiv:0806.0608 [gr-qc]; J. A. Gonzalez, F. S. Guzman and O. Sarbach, arXiv:0806.1370 [gr-qc]; D. H. Correa, J. Oliva and R. Troncoso, JHEP 0808, 081 (2008).

[19] N. Dadhich, S. Kar, S. Mukherjee and M. Visser, Phys. Rev. D 65, 064004 (2002); M. Cataldo, P. Salgado and P. Minning, Phys. Rev. D 66, 124008 (2002).

[20] L. A. Gergely, Phys. Rev. D 65, 127503 (2002); S. A. Hayward, Phys. Rev. D 65, 124016 (2002); H. Ellis, J. Math. Phys. 14, 104 (1973); K. A. Bronnikov, Acta Phys. Polonica B 4, 251 (1973).

[21] S. V. Sushkov and Y. Z. Zhang, Phys. Rev. D 77, 024042 (2008); S. V. Sushkov and S. W. Kim, Gen. Rel. Grav. 36, 1671 (2004).

[22] C. Barcelo, L. J. Garay, P. F. Gonzalez-Diaz and G. A. Mena Marugan, Phys. Rev. D 53, 3162 (1996). 